\title{
Optimized SCMA Codebook Design Using Rotation Based Sub-Divided Constellation for Overloaded System
}

Madhura K ( $\square$ madhura.k00@gmail.com )

Vignan's Foundation for Science Technology \& Research https://orcid.org/0000-0002-0105-855X

M.S.S. Rukmini

Vignan's Foundation for Science, Technology, and Research

Rajeshree Raut

Government College of Engineering

\section{Short Report}

Keywords: $5 \mathrm{G}$ wireless communication, LDPC, MPA, NOMA, SCMA, SER.

Posted Date: June 29th, 2021

DOI: https://doi.org/10.21203/rs.3.rs-598942/v1

License: (c) (i) This work is licensed under a Creative Commons Attribution 4.0 International License.

Read Full License 


\section{Abstract}

5G in wireless communication aims at deploying massive connectivity. Sparse Code Multiple Access (SCMA), proves to be an emerging candidate with multidimensional codebooks proposing, high shaping gain and advanced multiuser detection. A framework for designing a codebook for $200 \%$ overloaded SCMA system with system model is presented in this paper. This article aims at delineating a codebook by subdividing the Mother Constellation (MC) and rotating it to achieve a better Symbol Error Rate (SER) performance over higher values of SNR. Different Codebook designs are taken into consideration for comparing with the sub-constellation based $200 \%$ overloaded SCMA. Abstract $-5 \mathrm{G}$ in wireless communication aims at deploying massive connectivity. Sparse Code Multiple Access (SCMA), proves to be an emerging candidate with multidimensional codebooks proposing, high shaping gain and advanced multiuser detection. A framework for designing a codebook for $200 \%$ overloaded SCMA system with system model is presented in this paper. This article aims at delineating a codebook by subdividing the Mother Constellation (MC) and rotating it to achieve a better Symbol Error Rate (SER) performance over higher values of SNR. Different Codebook designs are taken into consideration for comparing with the sub-constellation based $200 \%$ overloaded SCMA.

\section{Full Text}

This preprint is available for download as a PDF. 